Goldschmidt 2021 Abstract

https://doi.org/10.7185/gold2021.5009

\title{
Soil organic matter dynamics post-fire
}

\section{ASMERET ASEFAW BERHE}

University of California, Merced

Presenting Author: aaberhe@ucmerced.edu

Fire, erosion, and soil carbon (C) dynamics overlap in space and time. Increased rates of erosion typically follow wildfires, and fire-altered or pyrogenic $\mathrm{C}$ (PyC, also referred to as black carbon) is redistributed vertically within soil profiles and laterally to lower landform positions along hillslopes, changing soil's C sequestration trajectory. Using case studies from wildfires that occurred in the Sierra Nevada Mountains (USA) I will discuss how the composition and magnitude of PyC redistributed by erosion varies considerably depending on fire severity and geomorphology of the landscape. Furthermore, our work demonstrates how PyC's hydrologic interactions determine: the amount that will enter the soil profile and experience microbial and geochemical alterations, whether it will be buried in depositional environments and stored on the landscape, or if it will be transported to streams and eventually to the ocean. This crucial first interaction with the hydrologic cycle occurs on the timescale of days to weeks, and therefore supersedes microbial decomposition as the primary control on charcoal's environmental persistence. 